\title{
Acoustic velocity and attenuation coefficient of magnetorheological fluids under electromagnetic fields
}

\author{
Min Shen ${ }^{1, \mathrm{a}, \mathrm{b}}$, Qibai Huang ${ }^{2, \mathrm{a} *}$ \\ ${ }^{a}$ State Key Laboratory of Digital Manufacturing Equipment and Technology, Huazhong University of Science and \\ Technology, 430074 Wuhan, China. \\ ${ }^{b}$ School of Mechanical Engineering and Automation, Wuhan Textile University, 430073 Wuhan, China;
}

\begin{abstract}
:
Magnetorheological fluid (MRF) is a class of smart material whose acoustic properties can be varied rapidly and reversibly by the applied magnetic field. The MRF is proposed to be as actively sound barriers or acoustical metamaterial. This paper presents a theoretical model to study acoustic propagation in MRF under fields based on the Biot-Stoll model.The model considers the coupling interaction between ferro particle and base fluid. This paper investigated the acoustic velocity and attenuation of a commercial MRF dependence on the different parameters such as carrier fluid viscosity, permeability and intensity of magnetic field. The calculated results show that the attenuation is increased with small field strengths and independent on field strength when the magnetization begins to saturate.
\end{abstract}

Keywords: Magnetroheological fluid, Biot-Stoll model, rheological model, attenuation coefficient, electromagnetic field.

\section{Introduction}

Both Electorrheological fluid(ERF) and magnetorheological fluid(MRF) are "smart materials" whose mechanical properties exhibit fast strong and reversible changes under electro or magnetic fields, which resulting in drastic changes in acoustic properties. Recently, the ERF and MRF are proposed to be as actively sound barriers or acoustical metamaterial[1-3]. Controlling applied voltage or magnetic field may enable more advanced acoustic capabilities such as cloaking or super-lensing[2-3]. However, ER material exhibits a number of shortcomings compared to the MR material including low yield strength, requirement of high voltage and greater sensitivity to common impurities. Hence, this paper is focused on MRF.

The MRF consists of micron size magnetically permeable particles suspended in a carrying liquid such as the mineral or silicon oil, as shown in Fig.1. It is can be seen from Fig1.a that the ferromagnetic particles are scattered randomly in the liquid carrier in the absence of magnetic fields. Fig. $1 \mathrm{~b}$ shows that a magnetic dipole moment is induced particles tend to align chain-like structures under magnetic fields, giving rise to significant variation in acoustic properties.

*Corresponding author:Tel.+8613071284580

E-mail ddress:qbhuang@mail.hust.edu.cn(Qibai,Huang)

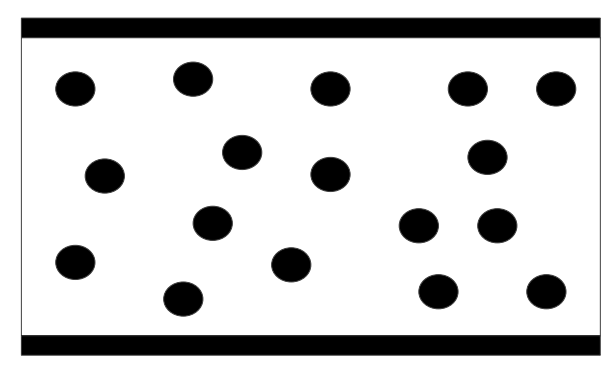

(a) No magnetic field

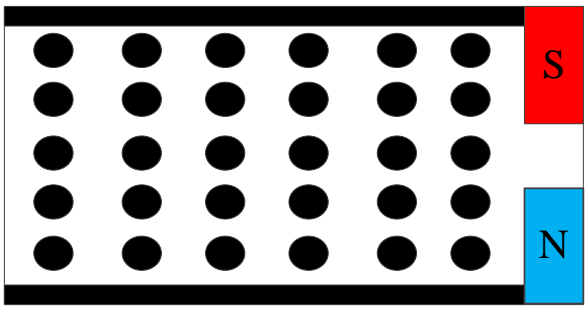

(b) Applied Magnetic field $\mathrm{H}$

Fig.1. The MR effect:(a) the particles in the absence of magnetic fields;(b) particles magnetized and form columns when applied field;

Fig.1. The MR effect:(a) the particles in the absence of magnetic fields;(b) particles magnetized and form columns when applied field;

The acoustic properties of MRF are very complex under magnetic field. Since discovery of the MRF, several theoretical and experimental studies have been conducted to investigate the acoustic properties in MRF.

Malinnovsky et al.[2-3] considered MRF as a linear 
chain consisting of the effective mass-spring oscillator. In their study, the theoretical model of acoustic wave propagation in MRF is based on one dimensional wave equation in the elastic medium.

Howarth et al.[4] conducted a series of acoustic experiments at the National High Magnetic Field Laboratory in Tallahassee, Florida. The acoustic sound speed of MR fluids was measured as functions of applied magnetic field strength, normal and orthogonal field orientations, and acoustic frequency. Their presentation discussed measurement methodology and preliminary results about the MRF.

Bramantrya et al.[5-6] focused on the experimental measurement of ultrasonic propagation velocity in the MRF change with an external magnetic fields intensity, interval time and angle of the magnetic field. They measured ultrasonic propagation velocity in MRF precisely and the clustering structures of MRF were analyzed in terms of elapsed time dependence, effect of external magnetic field strength and angel. A comparison of ultrasonic velocity propagation between magnetic and MRF was discussed.

Lo'pez et al.[7] measured ultrasonic acoustical velocity of a commercial MRF in static conditions(when the fluid is not flowing). In their experimental study, sound velocity and wave amplitude at $1 \mathrm{MHz}$ were analyzed as a function of the uniformity, direction and intensity of the magnetic field. The anisotropy and rearrangement micro-structure of MRF was analyzed in terms of sound speed and amplitude changes.

Previous theoretical models [2-3] study the sound propagation in MRF based on the one-dimensional wave equation of the elastic medium. However, coupling interaction of the ferromagnetic particle to the base fluid is not considered in present research. Hence, we propose a more accurate theoretical model to study acoustic propagation in MRF based on the Biot-Stoll theory which considers the coupling interaction of ferro particle skeletal frame to the base fluid. The other researchers [4-7] are restricted to the experimental investigation qualitative analysis the sound velocity and attenuation in MRF by ultrasonic technique. The sound propagation in relation to the inner structures of MRF and magnetic field is still not clear. Hence, this paper presents an analytical model for wave propagation in MRF based on Biot-Stoll theory, which has been developed for porous elastic media saturated by a compressible fluid[8-10]. In order to predict the effective material behavior of the composite, the MRF is regarded as homogeneous on macro scale. Then, we submit a new rheological model for description non-linear material effect of MRF under magnetic field. The flow viscosity is modeled as function of the shear rate under magnetic field. In Section 4, we discuss the corresponding numerical calculations for the acoustic attenuation and velocity relation on its viscosity, permeability inner properties and magnetic field.

\section{The sound wave propagation in MRF}

In presence of an external magnetic field, MRF is composed of assemblage ferromagnetic grains, which is the skeletal frame, and base fluid saturating the pores, which is the pore fluid. So the structure of MRF behaves as a skeletal frame filled with a base fluid, suggests an attempt of modeling its mechanical behavior by the Biot-Stoll theory.

Biot developed a comprehensive model of acoustic wave propagation in porous, elastic, solid medium saturated by a viscous fluid. In order to predict the acoustic properties of unconsolidated granular sediments. Stoll extended the Biot's model by considering friction between the grains by making the frame bulk and shear modulus complex. Nowadays, this extended Biot model is called the Biot-Stoll model. In Biot-Stoll model, there existences of two longitudinal waves in the medium and a shear wave at the interface of the medium. One of two longitudinal waves is called "the first kind". The other longitudinal wave is called "the second kind". The "first kind" is comparable to the usually observed longitudinal wave in a homogeneous medium (frame and fluid oscillate essentially in phase). In contrast, the longitudinal wave of the "second kind" is highly attenuated (frame and fluid oscillate essentially in anti-phase).

Only the first kind is important in MRF. The wave of the second kind is highly damped, because the oscillations of frame and fluid are nearly out of phase. This wave can be omitted. For the shear wave will not propagate in fluid state. In the solid state, a shear wave might exist, but because of the dominant fluid character 
will not become applicable and is omitted here, too.

In this study, we will treat only the first kind of longitudinal wave. Let $u$ is displacement of the frame, $\mathrm{U}$ is displacement of the pore fluid relative to the frame. Then $e$ is the dilation of a volume element attached to the frame and $\zeta$ is the relative dilatation between the frame and the fluid is expressed by

$$
\begin{aligned}
& e=\operatorname{div}(u) \\
& \zeta=\beta \operatorname{div}(u-U)
\end{aligned}
$$

where $\beta$ is the porosity.

The wave equations for the longitudinal wave in the porous saturated media derived by Biot are expressed as follows [8-9]:

$$
\begin{aligned}
& \nabla^{2}(H e-C \zeta)=\frac{\partial^{2}}{\partial t^{2}}\left(\rho e-\rho_{f} \xi\right) \\
& \nabla^{2}(C e-M \zeta)=\frac{\partial^{2}}{\partial t^{2}}\left(\rho_{f} e-\rho_{c} \xi\right)-\frac{\eta}{B_{0}} \frac{\partial \zeta}{\partial t}
\end{aligned}
$$

where the $\eta$ is fluid viscosity, the $B_{0}$ is the dynamic permeability of the skeletal frame, $\rho$ is the total density of the volume element of composite material $\rho=(1-\beta) \rho_{r}+\beta \rho_{f}, \quad \rho_{f}$ is density of the fluid

material and $\rho_{r}$ is the density of the solid grains.

Most of these authors calculate the added mass factor $\rho_{\mathrm{c}}$ is expressed as follows:

$$
\rho_{c}=\frac{\rho_{f}}{\beta} \gamma
$$

where $\gamma$ is the structure factor account for the apparent increase in fluid inertia caused by the tortuosity of the pores and $\beta$ is the porosity.

The complex parameters $H, C$ and $M$ describe the elastic porous medium and can be obtained from the complex elastic module of the components by

$$
H=\frac{\left(K_{r}-K_{b}\right)^{2}}{D-K_{b}}+K_{b}+\frac{4}{3} G_{b}
$$

$$
\begin{aligned}
& C=K_{r} \frac{K_{r}-K_{b}}{D-K_{b}} \\
& M=\frac{K_{r}^{2}}{D-K_{b}} \\
& D=K_{r}\left[1+\left(\frac{K_{r}}{K_{f}}-1\right) \beta\right]
\end{aligned}
$$

where $K_{f}$ is the bulk modulus of the fluid: $K_{r}=K_{r}{ }^{\prime}-i K_{r}^{\prime \prime}$ the bulk modulus of the solid material composing the porous frame(the bulk modulus of the individual particles in the case of granular media); $K_{b}=K_{b}{ }^{\prime}-i K_{b}^{\prime \prime}$ the bulk modulus and $G_{b}=G_{b}^{\prime}-i G_{b}^{\prime \prime}$ the shear modulus of the assemblage of particles measured for the case of constant pressure of the fluid in the pores.

For the MRF description, the fluid bulk modulus $K_{f}$ can be assumed purely elastic too. Different is the particle bulk modulus $K_{r}$, since there is no compact silicate material but carbonyl iron powder which is created directly in the silicone oil. Thus, the particles probably do not have a compact shape; more likely they consist of long, entangled molecules undergo structural rearrangement under load. In this paper this behavior is taken into account by a complex bulk modulus of the grain material.

Below a frequency limit which is indicated by pore size and viscosity, only Poiseuille flow exists in the composite material. Considering laminar flow of a fluid in a circular tube. Stoll[10] derived a function $F(\kappa)$ which accounts for the deviation from Poiseuille flow at higher frequencies. To do this, $\eta$ in Eq.(3) has to be replaced by $F(\kappa) \eta$. This has been detailed in many Biot-Stoll [8-10] theory applications and shall not repeat here.

This means that an additional or even artificial structure constant is introduced. Hovem et al.[11-12] 
have used another method. By inserting the flow correction function $F(\kappa)=F(\kappa)^{\prime}+i F(\kappa)^{\prime \prime}$ into Eq. (3) follows

$$
\begin{gathered}
\nabla^{2}(C e-M \zeta)=\frac{\partial^{2}}{\partial t^{2}}\left(\rho_{f} e-\rho_{c} \xi\right)-\frac{\eta F(\kappa)^{\prime}}{B_{0}} \frac{\partial \zeta}{\partial t} \\
\rho_{c}=\frac{\rho_{f}}{\beta}\left(1+\frac{\eta \beta}{B_{0} \rho_{f}} \frac{F^{\prime \prime}(\kappa)}{\omega}\right)
\end{gathered}
$$

The complex correction factor $F(\kappa)$, which is given by

$$
F(\kappa)=\frac{\kappa T(\kappa)}{4[1+2 i T(\kappa) / \kappa]}
$$

where $T(\kappa)$ is given by the complex Kelvin function

$$
T(\kappa)=\frac{\operatorname{ber}^{\prime}(\kappa)+\operatorname{ibei}^{\prime}(\kappa)}{\operatorname{ber}(\kappa)+\operatorname{ibei}(\kappa)}
$$

with $\kappa=a_{p}\left(\omega \rho_{f} / \eta\right)^{1 / 2}$

where $\eta$ is viscosity, $\rho_{f}$ is the fluid density, and $a_{p}$ is the pore size parameter.

Hovem and Ingram[11] have additionally calculated the pore size parameter $a_{p}$ and the permeability $B_{0}$ as

$$
a_{p}=\frac{d_{m}}{3} \frac{\beta}{\beta-1}
$$

$d_{m}$ is the average particle diameter, and

$$
B_{0}=\frac{d_{m}^{2}}{36 k^{\prime}} \frac{\beta^{3}}{(\beta-1)^{2}}
$$

Here the parameter $k^{\prime}$ should take into account the shape of the pores. This can be considered another artificial parameter.

To obtain frequency equation, solution for $e$ and $\zeta$ of the form

$$
\begin{aligned}
& e=A_{1} \exp [i(\omega t-l x)] \\
& \zeta=A_{2} \exp [i(\omega t-l x)]
\end{aligned}
$$

In Eqs.(15) and (16), $l=l_{r}+i l_{i}$ denotes the complex wave number for the longitudinal wave, $\omega$ is circular frequency and $x$ is the propagation distance. Upon transformation to the frequency domain, Eqs.(2)-(3) yields the longitudinal wave dispersion relation

$\left|\begin{array}{cc}H l^{2}-\rho \omega^{2} & \rho_{f} \omega^{2}-C l^{2} \\ C l^{2}-\rho_{f} \omega^{2} & \rho_{c} \omega^{2}-M l^{2}-i \omega F \eta / B_{0}\end{array}\right|=0$

The roots of Eq.(17) give the longitudinal wave velocities $c_{l}=\omega / l_{r}(\mathrm{~m} / \mathrm{s})$ and the attenuation coefficient $\alpha=l_{i}(\mathrm{~Np} / \mathrm{m})$ as a function of frequency.

\section{A new rheological model for MRF under electromagnetic fields}

The MRF demonstrates non-linear material behavior when an external electromagnetic field is applied. The rheological behavior of the MRF can be separated into two distinct pre- and post-field regimes. The Bingham plastic constitutive model has been extensively used to predict the post-yield behavior of MRF[13-14]. However, the MRF experiences shear thinning or shear thickening under electromagnetic field, the Bingham plastic model is not an accurate predictor for rheological behavior [15-16]. When the MRF undergoes a shear thinning or thickening effect, its rheological property is non-linear. This effect requires a fluid model to account for non-Newtonian behavior of MRF in the post-yield regime. Resiga[16] has investigated the rheological behavior of the MRF-132DG suspension using a rheometer Physica MCR300 with plate-plate magneto-rheological cell.

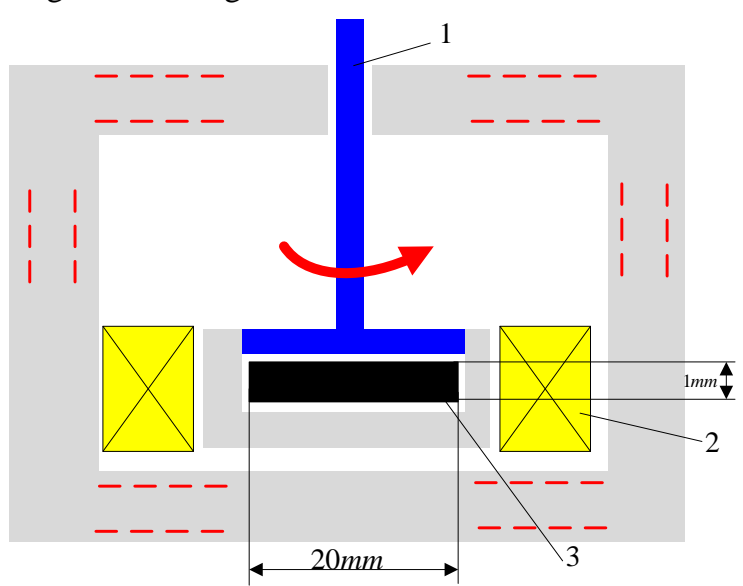

Fig.2. Experimental system of MRF rheological properties(1) Rotor. (2) coil. (3) MRF sample 
We propose a new rheological model for description post-yield shear thinning or thickening. A detailed description of the experimental system and the measuring process is shown in Fig.2. The viscosity-shear rate curves at uniform magnetic fields are measured using rheometer MCR301 as shown in Fig.2. The diameter and the gap of the parallel-plate system were $20 \mathrm{~mm}$ and $1 \mathrm{~mm}$, respectively. All the measurements are performed at room temperature. An electromagnet consisting of two refrigerated coil face to face has been used as a magnetic field source. The uniform magnetic fields are obtained with the advantage that the intensity of the magnetic field is easily varied by increasing or decreasing the current without change the field geometry. The magnetic field is measured with a Gauss meter FH51 provided by Magnet-Physik, placing the hall probe in different positions along the test cell when the magnetic fields are applied and no fluids are being tested.

As a result, the mathematical model to be used for fitting the experimental data of the MRF samples as:

$$
\tau(\dot{\gamma})=\tau_{N}(\dot{\gamma}) W_{1}(\dot{\gamma})+\tau_{H B}(\dot{\gamma}) W_{2}(\dot{\gamma})
$$

where the $\dot{\gamma}$ is the shear rate, $W_{1}(x)$ and $W_{2}(x)$ are weighting functions.

The Newtonian behavior $\tau_{N}$ is given by

$$
\tau_{N}(\dot{\gamma})=\eta_{0} \dot{\gamma}
$$

and the shear thinning behavior is described by the Hershel-Bulkley model

$$
\tau_{H B}(\dot{\gamma})=\tau_{0}+\tau_{1} x^{1-n}
$$

where $x=\dot{\gamma} / \dot{\gamma}^{*}, \dot{\gamma}^{*}$ is the characteristic shear, at the characteristic shear rate value, the rheological behavior corresponding to shift from quasi-Newtonian to shear thinning.

For the present analysis we have chosen

$$
\begin{aligned}
& W_{1}=1-\tanh (x) \\
& W_{2}=\tanh (x)
\end{aligned}
$$

Substituting Eqs(21)-(22) into Eq.(18), then

$$
\tau=\eta_{0} \dot{\gamma}[1-\tanh (x)]+\left[\tau_{0}+\tau_{1}(x)^{1-n}\right] \tanh (x)
$$

The shear stress vanishes as $\dot{\gamma} \rightarrow 0$, while the viscosity $\eta \equiv \tau / \dot{\gamma}$ has the limit value for vanishing shear rate

$$
\eta^{*} \equiv \lim _{\dot{\gamma} \rightarrow 0} \eta=\eta_{0}+\frac{\tau_{0}}{\dot{\gamma}^{*}}
$$

Eq.(24) clearly reveals that the start-up viscosity $\eta$

\begin{tabular}{|c|c|c|c|}
\hline Parameters & Symbol(Unit) & ERF & MRF \\
\hline $\begin{array}{l}\text { Solid } \\
\text { density }\end{array}$ & $\rho_{r}\left(k g / m^{3}\right)$ & 1260 & 3160 \\
\hline $\begin{array}{l}\text { Fluid } \\
\text { density }\end{array}$ & $\rho_{f}\left(k g / m^{3}\right)$ & 920 & 1000 \\
\hline $\begin{array}{c}\text { Grain bulk } \\
\text { modulus }\end{array}$ & $K_{r}^{\prime}\left(N / m^{2}\right)$ & $1.51 \times 10^{9}$ & $1.26 \times 10^{11}$ \\
\hline $\begin{array}{l}\text { Volumetric } \\
\text { Specitic loss }\end{array}$ & $\delta_{k r}$ & 0.4 & 0.4 \\
\hline $\begin{array}{l}\text { Fluid bulk } \\
\text { modulus }\end{array}$ & $K_{f}\left(N / m^{2}\right)$ & $9.8 \times 10^{8}$ & $9.8 \times 10^{8}$ \\
\hline $\begin{array}{c}\text { Grain } \\
\text { diameter }\end{array}$ & $d_{m}(\mathrm{~m})$ & $5 \times 10^{-5}$ & $10 \times 10^{-6}$ \\
\hline $\begin{array}{c}\text { Fluid } \\
\text { viscosity }\end{array}$ & $\eta\left(N s / m^{2}\right)$ & $5 \times 10^{-3}$ & $0.077 \sim 10^{6}$ \\
\hline $\begin{array}{c}\text { Frame shear } \\
\text { modulus }\end{array}$ & $\mathrm{G}_{b}^{\prime}\left(N / m^{2}\right)$ & $0 \sim 8 \times 10^{7}$ & $0 \sim 8 \times 10^{6}$ \\
\hline $\begin{array}{c}\text { Shear } \\
\text { specific loss }\end{array}$ & $\delta_{G b}$ & $0 \sim 2$ & $0 \sim 0.5$ \\
\hline $\begin{array}{c}\text { Frame bulk } \\
\text { modulus }\end{array}$ & $K_{b}^{\prime}\left(N / m^{2}\right)$ & $0 \sim 4 \times 10^{8}$ & $0 \sim 4 \times 10^{8}$ \\
\hline $\begin{array}{l}\text { Volumetric } \\
\text { specific loss }\end{array}$ & $\delta_{K b}$ & $0 \sim 2$ & $0 \sim 0.5$ \\
\hline Porosity & $\beta$ & 0.5 & $0.1 \sim 0.5$ \\
\hline Permeability & $B_{0}\left(m^{2}\right)$ & $4 \times 10^{-12}$ & $4 \times 10^{-12}$ \\
\hline
\end{tabular}
incorporate both the contribution from the Newtonian behavior $\eta_{0}$ and contribution from the yield stress of the

Hershel-Bulkley model $\tau_{0}$.

\section{Theoretical prediction of longitudinal wave velocity} and attenuation coefficient

Table.1 The Biot-Stoll Parameters of MRF-132DG 
This work studied the relation of the first kind longitudinal wave velocity and attenuation coefficient to the inner structures and the uniform electromagnetic field. Using the equations in the preceding section, the longitudinal wave velocity and attenuation coefficient of the first kind are calculated for MRF-132DG material. Table 1 lists the parameters of the model for the MRF investigated which is typical of MRF-132DG manufactured by Lord Corporation. All parameters of MRF in braces are either directly supplied by the manufacturer or have been calculated. The calculations for the linear equations are numerically performed. For the MRF description, the fluid bulk modulus $K_{f}$ can be assumed purely elastic, because in our case the base fluid is low-viscosity silicone oil.

We have tested the rheological behavior of the MRF-132DG by rheometer Physica MCR301. The magnetic field applied to the MRF was generated by the built-in coil of the MRF cell. Both oscillatory and rotational test have been performed. All tests have been performed for variable current from 0.25,0.5,0.75,1.0,1.5 to $2.0 \mathrm{~A}$ and the electromagnetic field intensity increases linearly from 80Tesla to 650Tesla .

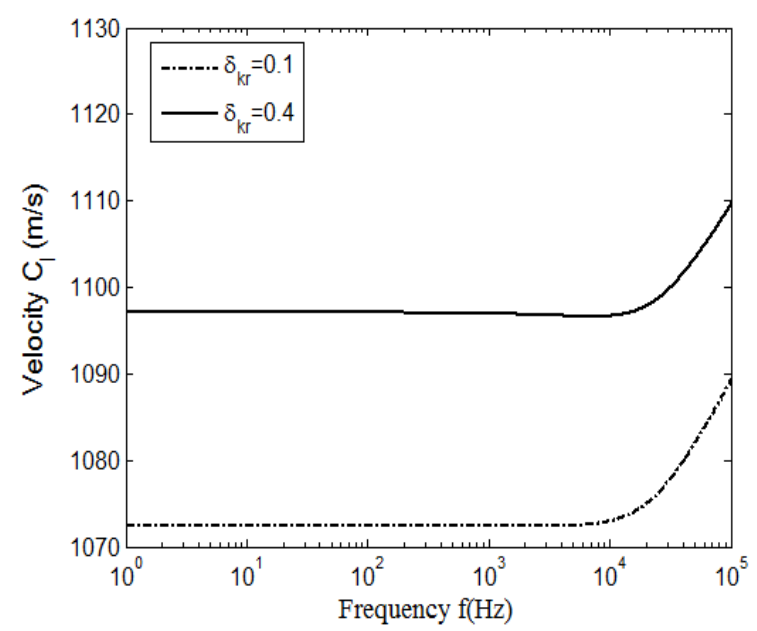

Fig.3. Semi-logarithmic plot longitudinal velocity calculated for the ERF

To verify the correctness of this model, the first kind longitudinal wave velocity and attenuation coefficient of the ERF are calculated. The parameters value of the ERF used are given in Table 1 reference Huang et al.[17]. Fig.3 shows the longitudinal velocity of the first kind as a function of frequency and Fig.4 shows attenuation coefficient as a function of frequency. The influence of external electro-field to acoustic properties of ERF is not considered in the study.

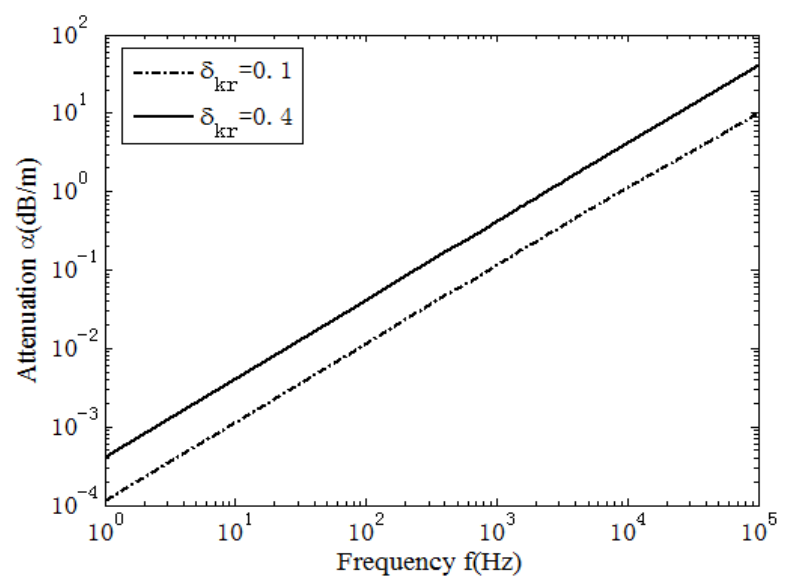

Fig.4. Logarithmic plot attenuation calculated for the ERF

Fig.5 shows the viscosity curves (dynamic viscosity VS. Shear rate) variation with the intensity of the electromagnetic field (proportional to the intensities of the electric current in the coil). It can be seen from Fig.5 that there is a substantial jump in viscosity values even applied a rather weak electromagnetic field, and a rapid saturation as the intensity is increased. In most of the experimental data reported in the MRF literature the viscosity diverges with a slope of -1 at low shear rates, and thus an apparent yield stress is invoked.

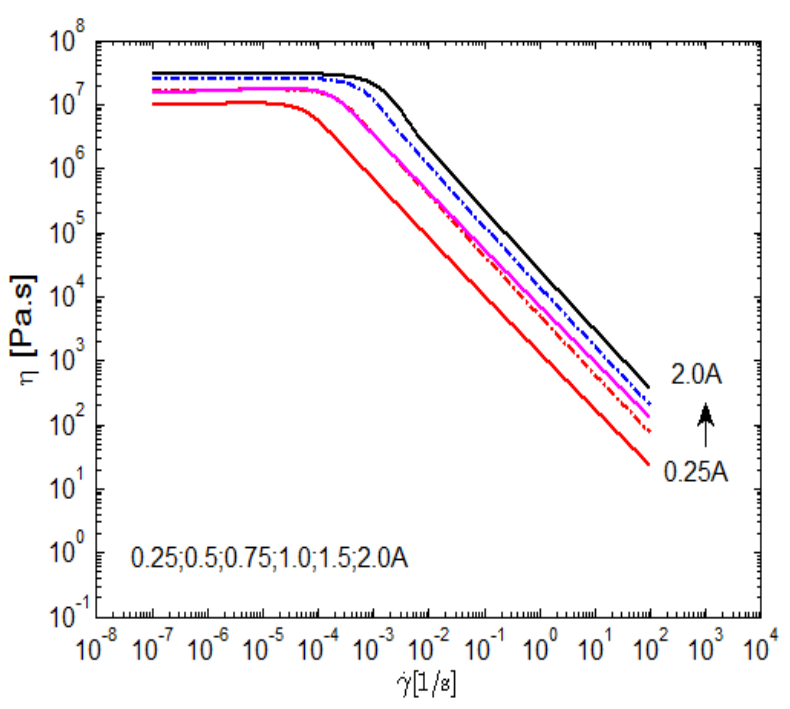

Fig.5. Viscosity curves for variable magnetic field strength

Fig.6 shows the magnetic intensity propotional with the intensity of the electric current in the coil. The 
magnetic field applied to the MRF was generated by the built-in coil as shown in Fig.2. The magnetic field lines perpendicular to the plate through the gap filled with MRF and the magnetic flux is measured by PF-035 Tesla meter. The magnetic flux density in the measuring gap depends not only on the coil current I, but also on the materials through which the flux is following, in particular, the magnetic properties of the MRF.

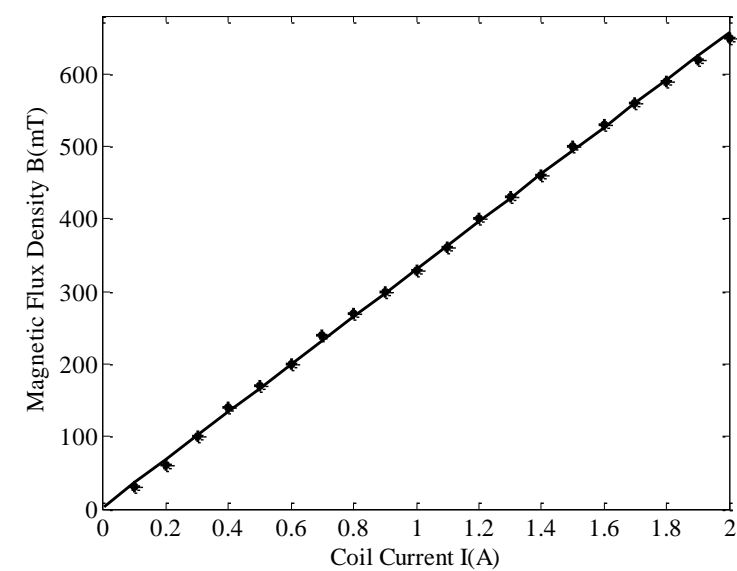

Fig.6. The dependence of the magnetic flux density on the driving coil current $I$ in the measuring gap filled with MRF-132DG.

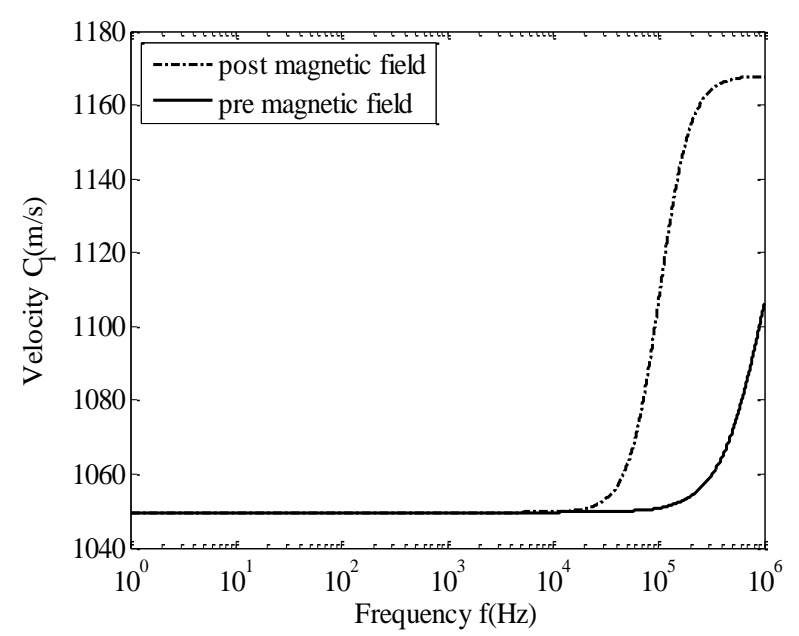

Fig.7. Comparison of longitudinal wave velocity of MRF pre-magnetic field and post-magnetic field

Fig.7 shows the longitudinal wave velocity of the first kind for MRF without magnetic field comparing with applied an external magnetic field. The MRF is initially fluid-like state without magnetic field $(I=0)$. The viscosity is about $0.077 \mathrm{~Pa} . \mathrm{s}$ in fluid-like state. Ferromagnetic particle changes to chian stucture in the presnece of a magnetic field, the vicinity of intergranular contacts is filled with base fluid.This characteristic causes that the viscosity is greater than $10^{3}$ Pas ( $I \geq 0.25 \mathrm{~A})$ in a few seconds. In this calculation, the other parameters are listed in Table.1. It can be seen from Fig.7 that longitudinal velocity of MRF under magnetic field is same as velocity without the magnetic field at lower frequency. But the longitudinal velocity starts to increase at higher frequency under a weak magnetic field. The reason is that the MRF is initial fluid-like state without magnetic field. In the presence of a magnetic field, the MRF changes from a viscous fluid to an almost solid body and the shear modulus changes by several orders of magnitude, so the longitudinal velocity of the first kind is increased by applied a weak magnetic field.

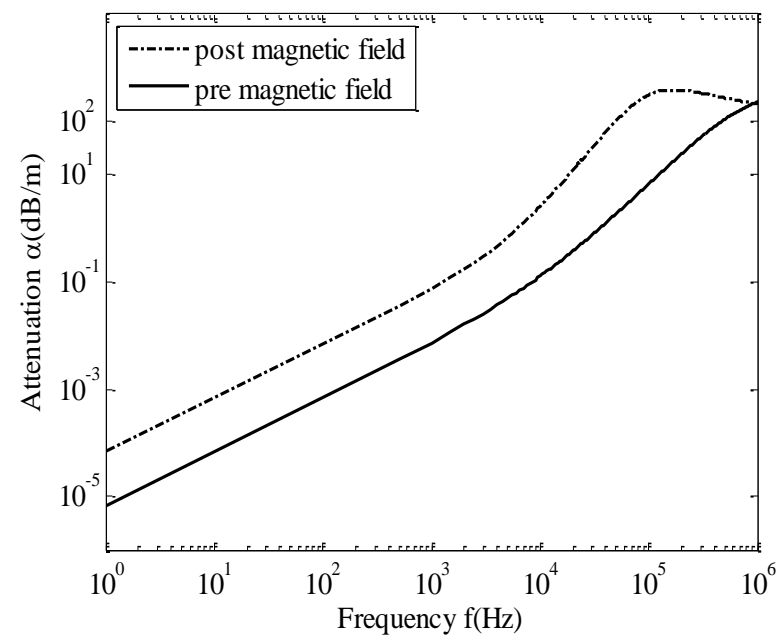

Fig.8. Comparison of attenuation coefficient of MRF pre-magnetic field and post-magnetic field

Fig.8 shows attenuation coefficient of MRF without magnetic field comparing with applied magnetic field. It can be seen from Fig. 8 that the attenuation coefficient increases under magnetic field. The skeletal frame exists without the magnetic field, but it shows negligible values of shear and bulk modulus. When placed in magnetic field, the structure of MRF behaves as a skeletal frame filled with a base fluid, the skeletal frame cause viscoelastic deformation. When the wave is incident in the MRF, the viscosity force of the base fluid and the interaction friction force between the particle and base fluid could attenuate the sound energy to the thermal energy. So the viscous loss could increase longitudinal wave attenuation. 
Fig.9 shows the three-dimensional representations of longitudinal wave velocity in the frequency of $1 \mathrm{~Hz}$ to $1 \mathrm{MHz}$, and the viscosity range of $0.077 \mathrm{~Pa}$.s to $10^{2} \mathrm{~Pa}$.s. When placed in a weak magnetic field, the viscosity of MRF trends to saturation above $10^{3} \mathrm{~Pa}$.s. It is seen from Fig.9 that the longitudinal wave velocity of the first kind increases dependent on the field strength, but when the viscosity above the 60 , the wave velocity is nearly kept $1180 \mathrm{~m} / \mathrm{s}$ and independent on frequency. Because the conventional MRF is initially as fluid-like without magnetic field, the viscosity is low and the longitudinal wave velocity of the first kind is slow. When apply an external magnetic field, the MRF is as solid-like. Hence, the longitudinal wave speed in the solid state is faster than the initial fluid state.

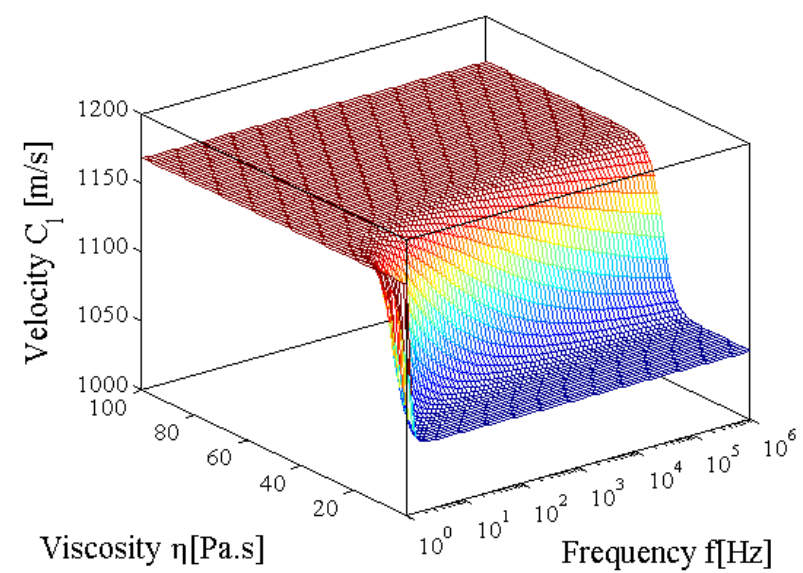

Fig.9. Three-dimensional representation of the longitudinal wave velocity as functions of viscosity and frequency for MRF

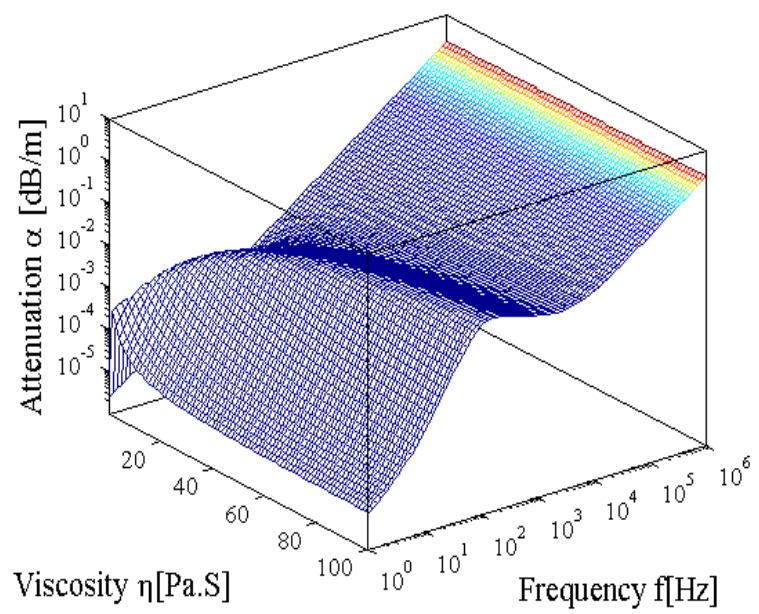

Fig.10. Three-dimensional representation of the attenuation as functions of viscosity and frequency for MRF
Fig.10 shows the three-dimensional representations of attenuation coefficient variation changes with frequency and viscosity. It is seen from Fig.10 that the attenuation coefficient is very complex dependent on both frequency and viscosity.There is a sharp jump on the curve below viscosity $20 \mathrm{~Pa} . \mathrm{S}$ then the attenuation coefficient is decreased after the viscosity above $20 \mathrm{~Pa} . \mathrm{S}$ below $10^{3} \mathrm{~Hz}$. Because the particles are aggregated by magnetic field which makes the friction increase between fluid and aggregate surface provide more attenuation compared with the initial suspension of non-aggregated particles. On the other hand, the increase in magnetic field strength causes variations of the microstructure. When the ferro-particles are aligned with the acoustic propagation, the sound attenuation is increased to maximum value.

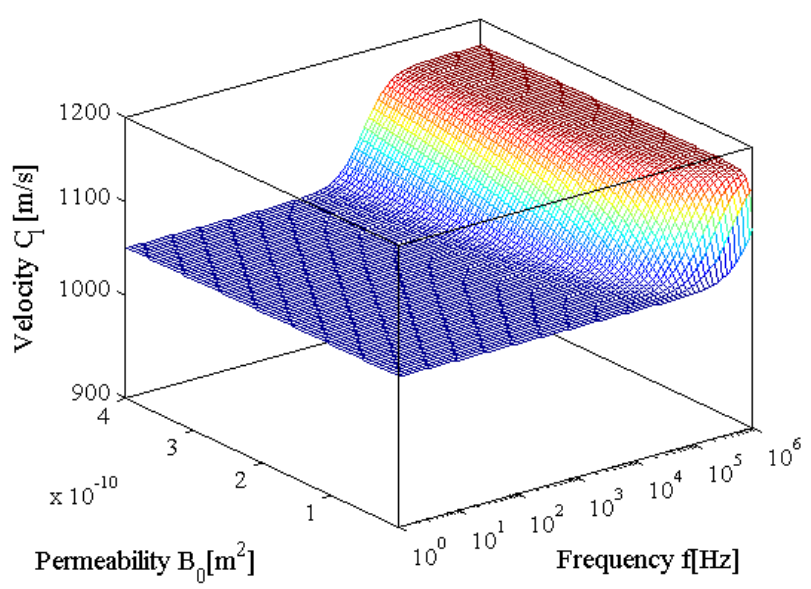

Fig.11. Three-dimensional representation of the longitudinal wave velocity as functions of permeability and frequency for MRF

Fig.11 shows the three-dimensional representations of longitudinal wave velocity variation with frequency and permeability. The magnetic field is not only responsible for the reorientation of particles but it also makes the chain skeleton become rigid with increasing intensity and the permeability also changes. Because of the small fluid friction damping, there is a small amount of dispersion in longitudinal wave of the first kind. The pattern of this dispersion is shown in Fig.11. It is seen from Fig.11 that the velocity increase occur over a rather narrow band of frequency. In low frequency, the longitudinal wave velocity of the first kind is almost no variation as the permeability changes.

Fig.12 shows the three-dimensional representations of 
attenuation coefficient variation with frequency and permeability. It is seen from Fig.12 that the attenuation coefficient increases as the permeability increases above the frequency of $10^{2} \mathrm{~Hz}$. The curves suggest highly nonlinear forms. Reason for this usually involves the inner ferro-particle interactions apply an external field. The interparticle magnetization interaction force depends on the external field.

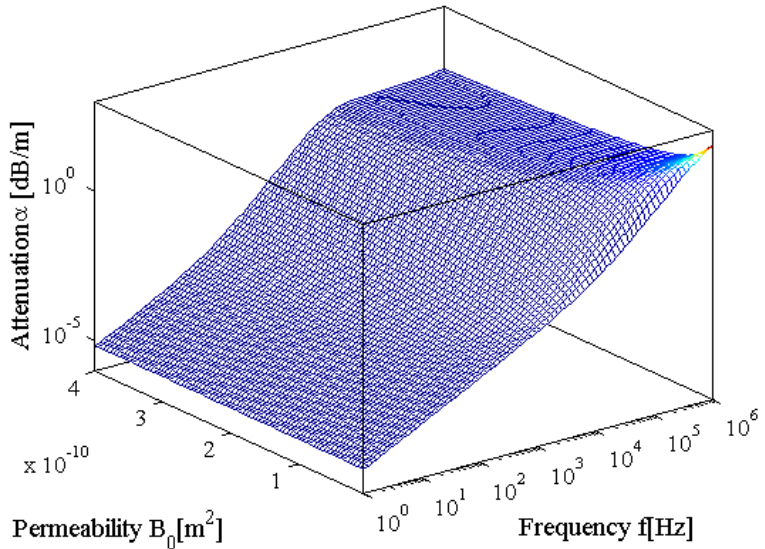

Fig.12. Three-dimensional representation of the attenuation as functions of permeability and frequency for MRF

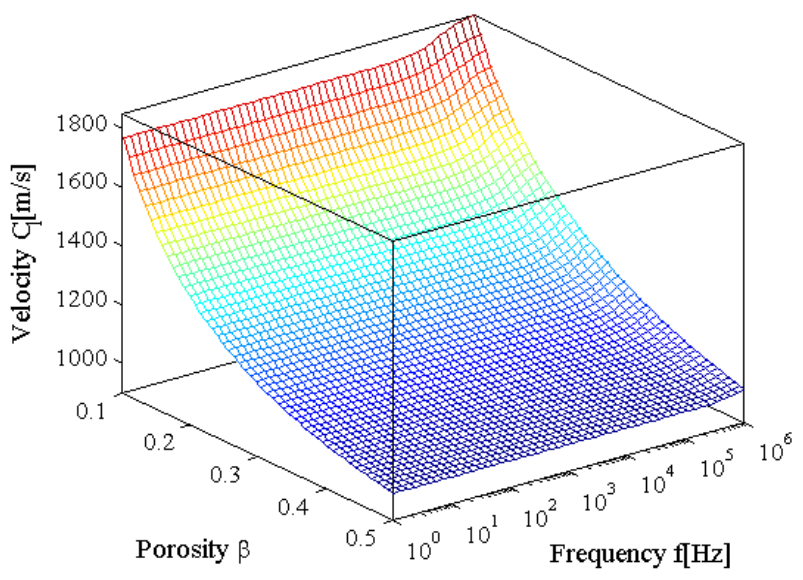

Fig.13. Three-dimensional representation of the sound speed as functions of porosity and frequency for MRF

Fig.13 shows the three-dimensional representations of sound speed variation changes with frequency and porosity. The MRF is similar to other aggregating suspensions, in the absence of an external magnetic field, the viscosity of MRF slightly increases when the volume fraction incrasing at low values and abruptly increases once the concentration is larger than a critical volume fraction $\phi_{c}[18]$. It is well known that in a colloidal suspension, only one longitudinal mode can exist when an elastic excitation propagates in it if the wavelength is much larger than the characteristic size of the suspended particles.In calculating the sound speed, we assume that in the first mode, the elastic perturbation propagates through channels of almost clear fluid. Provided that the perturbation wavelength is much larger than the characteristic size of the iron particles.

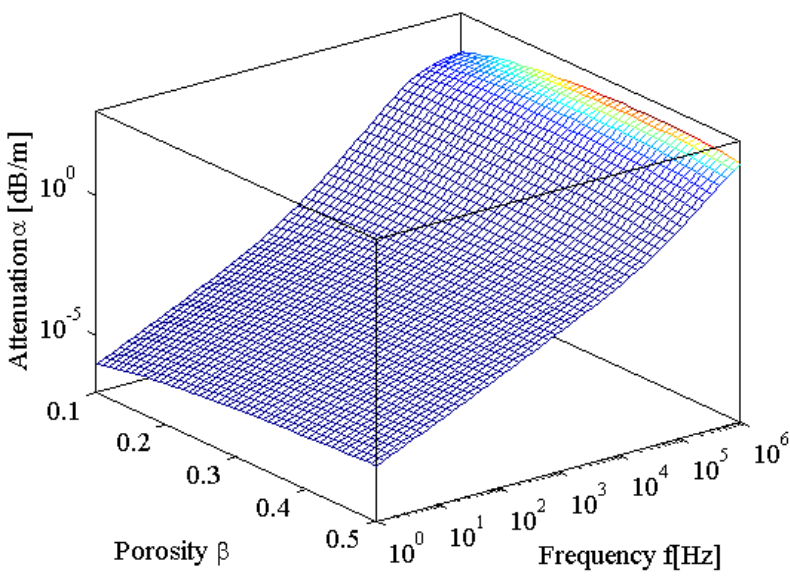

Fig.14. Three-dimensional representation of the attenuation coefficient as functions of porosity and frequency for MRF

Fig.14 shows the three-dimensional representations of attenuation coefficient variation changes with frequency and porosity. It is seen from Fig.14 that the attenuation coefficient is slightly increase as the porosity increasing at low values. The isolated clusters exsit below a cirtical volume fraction $\phi_{c}$, the viscosity is at low value, once the concentration is lager than cirtical volume fraction $\phi_{c}$ resulting in viscosity of MRF slightly inceased. On the other hand, the effect of magnetic field strength in MRF below $\phi_{c}$, initially the zero shear viscosity grows with the field strength up to a certain point and the loss factor also independent of the frequency.

\section{Conclusion}

In present paper, we propose a more accurate theoretical model to study acoustic velocity and attenuation relation on its viscosity, permeability inner properties and magnetic field. The acousitc propagaiton in MRF were modelded based on the Biot-Stoll theory for porous material. This acoustic theoretical model considerd the coupling interaction of ferro paritcle skeletal frame to the base fluid. Through numerical 
sinulation, the sound speed and attenuation coefficient can be got. The numerical results show that the magnetic field creats microstructures which are responsible for the increase in sound attenuation and the attenuation coefficients increase with the small field strengths, as the magnetization begins to satuate, the attenuation coefficients become independent on field strength.

\section{ACKNOWLEDGEMENTS}

The present research was supported by the China National Science Foundation (No.51175195, No.5155344). The magneto-rheological investigations were performed at State Key Laboratory of Digital Manufacturing Equipment and Technology, Huazhong University of Science and Technology, Wuhan,China.

The author is grateful to the referees for their useful comments.

\section{Reference}

[1]M.Szary, The analytical model of rheological fluid for vibration and noise control, Proceedings of Meetings on Acoustics, Montreal, Canada, 2013;19:1-8.

[2]V.S.Malinovsky and D.M.Donskoy, Electro-magnetically controlled acoustic metamaterials with adaptive properties, Journal of the Acoustical Society of America, 2012;132:2866-2872.

[3]D.M.Donskoy, V.S.Malinovsky, Broadband acoustic metamaterials with electro-magnetically controlled properties, Proceedings of Meetings on Acoustics, Montreal,Canada, 2013;19:1-8.

[4]T.R.Howarth,F.Fratantonio,J.E.Boisvert,A.Bruno,C.L. Scandrett,W.M.Wynn and P.S.Davis, Acoustic behavior of magnetorheological fluids in magnetic fields, Journal of the acoustical society of America, 2011;13:2359.

[5]M.A.Bramantya, M.Motozawa, H.Takuma, M.Faiz and T.Sawada, Experimental analysis of clustering structures in magnetic and MR fluids using ultrasound, 11the conference on electroheological fluids and magnetorhoelogical suspensions,2009,149:1-4.

[6]M.A.Bramantya,M.Motozawa and T.Sawada, Ultrasonic propagation velocity in magnetic and magnetorheological fluids due to an external magnetic field, Journal of Physics:Condensed Matter, 2010,22:1-5.
[7]J.R.Lo'pez, L.E.Segura and F.M.de.E. Freijo,Ultrasonic velocity and amplitude characterization of magnetorheological fluids under magnetic fields, 2012; 324: 222-230.

[8]M.A.Biot, Theory of propagation of elastic waves in a fluid-saturated porous solid, Part I:Low frequency range, Journal of the Acoustical Society of America, 1956; 28:168-178.

[9]M.A.Biot, Theory of propagation of elastic waves in a fluid-saturated porous solid, Part II:Higher frequency range, 1956;.28:179-191.

[10]R.D.Stoll, Acousitc waves in ocean sediments, Geophysics, 1977; 42(4):715.

[11]J.M.Hovem and G.D.Ingram, Viscous attenuation of sound in saturated sand, Journal of the Acoustical Society of America, 1979; 66:1087-1812.

[12]J.M.Hovem, Viscous attenuation of sound in suspension and high-porosity marine sediments, Journal of the Acoustical Society of America, 1980; 67:1559-1563.

[13]H.A.Barnes, J.F.Hutton and K. Walters, An introduction to rheology, 1st ed. Amsterdam: Elsevier, 1989.

[14]M.Obst, M.Kästner, F.Adam, J.Brummund and V.Ulbricht, Modelling the material behaviour of magnetorheological fluids under shear deformation,PAMM.Proc.Appl.Mech,2011;11:415416.

[15]A.G,Olabi and A.Grunwald, Design and application of magnetorheological fluids, Materials and Design, 2007;28:2658-2664.

[16]D.S.Resiga, A rheological model for magneto-rheological fluids, Journal of Intelligent Material Systems and Structures, 2009; 20:1001-1009.

[17]L.H.Ding,Q.B.Huang and Z.S.Xu, The energy reflection coefficient of electrorheological fluid with continuously changing acoustic impedance, Int J Mech Mater Des,2010;6:135-145.

[18]J.P.Segovia-Gutierrez, Nonlinear viscoelasticity and two-step yielding in magnetorheology: A colloidal gel approach to understand the effect of particle concentration, Journal of Rheology, 2012, 56 (6):1429-1448 